Pure Sciences

Poster

Abstract ID: 138

\title{
Hearing impairment among scuba divers in Kuantan and neighbouring regions
}

\author{
Marina L. Alisaputri ${ }^{\mathrm{a}}$ | Siti Hazwani bt Yusoh $^{\mathrm{b}}$ \\ ${ }^{a}$ Kulliyyah of Allied Health Sciences, International Islamic University Malaysia \\ ${ }^{b} J a b a t a n$ Otorinolaringologi, Hospital Sultanah Nur Zahirah, Kuala Terengganu, Terengganu
}

Introduction: Hearing impairment has been listed as one of the health risks associated with scuba diving. Evidence of hearing loss associated with inner ear barotrauma was found in previous studies. This cross-sectional study investigates the relationship between hearing status and years, depth and total hours of diving. Methods: Thirty male divers from Kuantan and neighbouring areas, with age ranging between 22 and 45 years old, were recruited as subjects. Details regarding diving activity were gathered using a set of questionnaires. Assessment of hearing status was done using otoscopic examination, tympanometry, pure tone audiometry (PTA) and distortion-product otoacoustic emission (DPOAE). Results: Fifteen subjects were found to have sensorineural hearing loss at high frequencies. Pearson's correlation showed no significant correlation between pure tone hearing thresholds and years, depth and total hours of diving. Significant negative correlation was found between DPOAE at $6 \mathrm{kHz}$ on the left ear and years of diving $(r=-0.378, p=0.04)$ and depth of diving $(r=-0.404, p=0.027)$. Conclusions: These findings indicate that scuba diving activity may affect cochlear function, particularly in the basal region of the cochlea.

KEYWORDS: hearing impairment, scuba diving, distortion product otoacoustic emission 